\title{
Pain Control and Quality of Life after Peripheral Neurectomy in Patients with Refractory Trigeminal Neuralgia
}

\author{
Kumar Nilesh
}

\begin{abstract}
Aim and objective: The present study was conducted to evaluate the efficacy of peripheral neurectomy (PN) in the management of trigeminal neuralgia (TN) not responsive to medical management.

Materials and methods: Patients diagnosed with refractory TN not fit for major surgery or not willing for the neurosurgical intervention were enrolled for the study. The success of the surgery was assessed by pain score in the postoperative period [measured by visual analog scale (VAS)]. Quality of life (QOL) of patients was evaluated using the Short-Form Health Survey (SF-36) tool consisting of eight domains, measuring both the physical and mental components of wellbeing. The statistical analysis was done by using IBM SPSS ${ }^{\circledR}$ software version 25 (IBM Corp., USA). The mean value and standard deviation for each of the parameters were considered and checked for statistical significance using the analysis of variance (ANOVA) test. The difference was considered significant if $p<0.05$.

Results: Thirty-five neurectomies were performed in 31 patients. Patients were on carbamazepine therapy for 6-60 months, with an average dose of $812.90 \mathrm{mg} /$ day. Mandibular division was most commonly involved ( $n=18 ; 58 \%)$, followed by maxillary $(n=7 ; 23 \%)$, and ophthalmic division $(n=2 ; 6 \%)$. The earliest recurrence of pain was seen at the sixth month in one patient. At the 1st year follow-up, poor and fair control of pain were seen in $3(10 \%)$ and $10(32 \%)$ patients, respectively. At the 3 years, recurrence was seen in $32.25 \%(n=10)$ patients. There was an improvement in QOL score immediately after PN, which despite the decrease on long-term follow-up, was considerably above the preoperative value.

Conclusion: Peripheral neurectomy is a simple, minimally invasive option for the management of refractory TN. Although recurrence is a limitation for its long-term success, it does help to achieve pain control and improve QOL.

Keywords: Carbamazepine, Conservative management, Peripheral neurectomy, Trigeminal neuralgia.

World Journal of Dentistry (2021): 10.5005/jp-journals-10015-1841
\end{abstract}

\section{INTRODUCTION}

Trigeminal neuralgia (TN) is a rare neurosensory disease of the orofacial region involving the fifth cranial nerve. It is defined as "unilateral disorder characterized by brief electric shock-like pain, abrupt in onset and termination, and limited to the distribution of one or more divisions of the trigeminal nerve". ${ }^{1}$ The disease is mostly idiopathic. Common etiological factors include compression of the nerve trunk due to central pathology/compression by tortuous vessel or demyelination of the involved nerve. ${ }^{2}$ Usually, there is the involvement of a single division of the trigeminal nerve, which may slowly spread to the other divisions. The characteristic features of pain in TN are spontaneous short episodes of triggered ipsilateral hemifacial pain, explained as sharpshooting, electric shock-like, stabbing, burning, crushing, or combination in nature. ${ }^{3}$ The pain is characteristically one-sided and restricted in the area of innervation of the trigeminal nerve. The pain is often so severe, that it significantly compromises the quality of life (QOL) of the affected individual.

The primary treatment for TN is medical therapy, carbamazepine being the drug of choice. ${ }^{4}$ However, medicinal therapy over a period of time may result in a relapse of pain leading to TN refractory to medicinal therapy. Long-term medical therapy is also associated with significant side effects, warranting its discontinuation. Surgical therapies can be offered to patients when medical therapy is ineffective or is associated with significant side effects. Numerous surgical procedures have been reportedly used for the management of TN with varying degrees of invasiveness, difficulty, and efficacy. ${ }^{5}$ Surgical management can broadly be divided into procedures at the Gasserian ganglion level (radiofrequency thermos-coagulation,
Department of Oral and Maxillofacial Surgery, School of Dental Sciences, Krishna Institute of Medical Sciences (Deemed to be University), Karad, Maharashtra, India

Corresponding Author: Kumar Nilesh, Department of Oral and Maxillofacial Surgery, School of Dental Sciences, Krishna Institute of Medical Sciences (Deemed to be University), Karad, Maharashtra, India, Phone: +91 9158542384, e-mail: drkumarnilesh@yahoo.com

How to cite this article: Nilesh K. Pain Control and Quality of Life after Peripheral Neurectomy in Patients with Refractory Trigeminal Neuralgia. World J Dent 2021;12(4):278-284.

Source of support: Nil

Conflict of interest: None

glycerol rhizolysis, balloon compression, stereotactic radiosurgery, and microvascular decompression) and peripheral procedures [peripheral neurectomy (PN), alcohol injections, cryotherapy]. ${ }^{6}$

Among the surgical procedures, PN is a simple, low-risk technique. In this procedure, the postganglionic peripheral portion of the involved branch of the trigeminal nerve is avulsed, as its exit from the foramina in its extracranial path. Peripheral neurectomy is relatively less invasive than the other surgical procedures and hence has low cost and morbidity associated with it. Although recurrence is the most common limitation of $\mathrm{PN}$, it can still be a useful treatment option, especially in a rural setting with limited resources. The present prospective clinical study was conducted to evaluate the efficiency of PN for the management of refractory $\mathrm{TN}$ in a rural, tertiary care center. 


\section{Materials and Methods}

The present prospective clinical study was conducted in patients with refractory $\mathrm{TN}$ reporting to the oral and maxillofacial surgery clinic, between February 2014 and January 2017. A total of 32 patients who agreed to participate and give written consent were included in the study, after clearance from the institutional ethical committee (342/2013-2014). Patients on previous oral carbamazepine (either as a single drug or combination), who had become refractory to the medical therapy or had severe side effects to it, were considered for the study. As per the department protocol, alternative pharmacological drugs were advised (including oxcarbazepine, baclofen, gabapentin, and pregabalin). On failure of the second and third line of drug therapy to provide pain relief or on intolerance to medicine or reluctance of patient to continue with medical management, surgical options were given to the patients.

Diagnosis of TN was based on Sweet's criteria, which included; paroxysmal and unilateral pain, provoked by light touch to the face (trigger zone), confined to trigeminal distribution, with no sensory deficit. ${ }^{7}$ Previous carbamazepine therapy with pain relief was also considered positive for the diagnosis of TN. Inclusion criteria to enroll patients for PN were:

- Patients with a previous history of carbamazepine therapy, which has failed to give desired relief from pain even after increased dosage.

- A severe side effect of long-term carbamazepine therapy, warranting its discontinuation.

- Patients with no obvious central pathology or vascular compression at the nerve root.

- Patients who were either not fit or willing for neurosurgical intervention.

- Patients where the pain could be localized to one or more terminal divisions of the trigeminal nerve.

The exclusion criteria were:

- Patients $<40$ years of age.

- Patients with atypical presentation and/or bilateral involvement.

- Known allergy to local anesthetic agents.

- Patients previously treated with neurectomy or any other surgical procedure.

All the patients fulfilling the above criteria and willing to participate in the study were enrolled. All the patients were subjected to magnetic resonance imaging to rule out the central lesion. Orthopantomogram and/or paranasal sinus view radiographs were advised to exclude any local cause or pathology.
Demographic details of the patient, duration, and dosage of carbamazepine therapy at the time of examination, affected, side along with the nerve involved were recorded. The terminal branch of the trigeminal nerve involved was identified by the area of localization of pain by the patient. Confirmation the same was achieved by diagnostic block with $2 \%$ lignocaine with 1:200,000 adrenaline. The patients were taken up for PN, either under local or general anesthesia. The surgical steps of the PN procedure included:

\section{Infraorbital Nerve Neurectomy}

Terminal branches of the infraorbital nerve were approached through an intraoral vestibular incision. The submucosal tissue was stripped in a subperiosteal plane over the anterior surface of the maxilla, superior to the infraorbital ridge. The intraorbital foramen was visualized. The nerve was carefully dissected and freed of its soft tissue attachments. The main truck of the nerve was held with a hemostat, as it exited the foramen, and the entire nerve was avulsed by reeling the hemostat. Bleeding from the foramen was controlled using electrocautery and/or bone wax. Closure of mucosal incision was done using resorbable sutures (Fig. 1).

\section{Inferior Alveolar Nerve Neurectomy}

The inferior alveolar nerve was accessed intraorally. A linear incision was placed over the anterior border of the ramus of the mandible. Soft tissue over the anterior and medial surface of the ramus was reflected in the subperiosteal plane. Careful blunt dissection was done medially at the level of the occlusal plane, to identify the inferior alveolar neurovascular bundle as it entered the mandibular foramen. The nerve was held in a hemostat. Two sutures were passed around the nerve, one above and one below the hemostat and tied. The nerve was resected between the two ligature sutures and the distal segment of the nerve was avulsed holding it by the tip of the hemostat. Closure of mucosa was one using resorbable sutures (Fig. 2).

\section{Mental Nerve Neurectomy}

The mental nerve was approached through an intraoral vestibular incision in the mandibular premolar region. Subperiosteal dissection was done to raise the flap over the mandible. Mental foramen was identified with the main trunk of the mental nerve. The terminal branches of the nerve were carefully dissected and freed from surrounding attachments. The main trunk of the nerve was held in a hemostat, close to the foramen and the nerve was avulsed. Hemostasis was attained and closure of mucosa was done using resorbable sutures (Fig. 3).
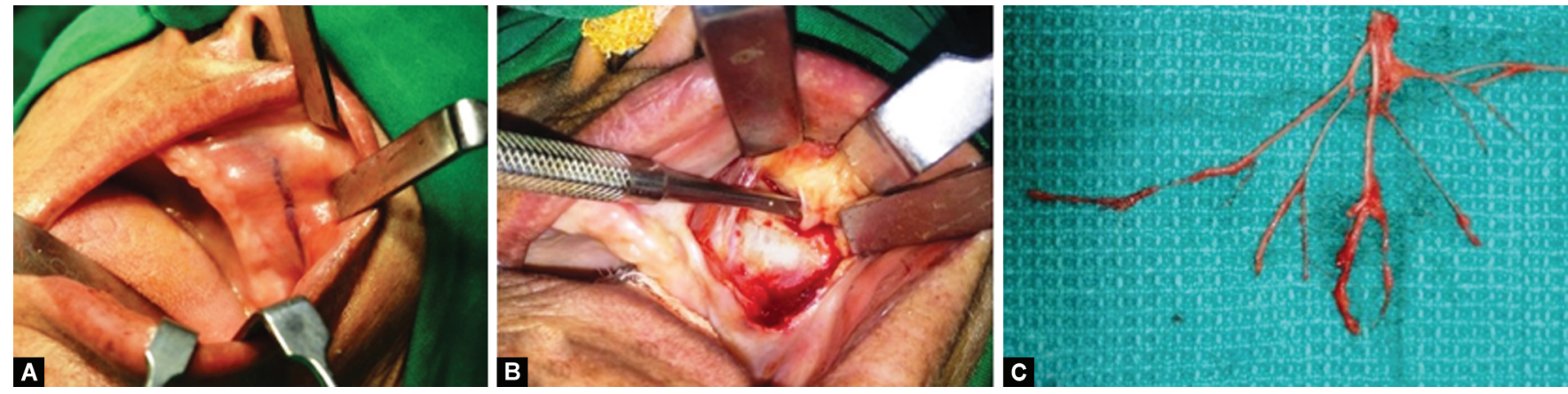

Figs $1 \mathrm{~A}$ to $\mathrm{C}$ : Intraoperative photographs of infraorbital nerve neurectomy showing the incision marking at upper buccal vestibule (A), dissection and exposure of the nerve (B), and avulsed nerve (C) 


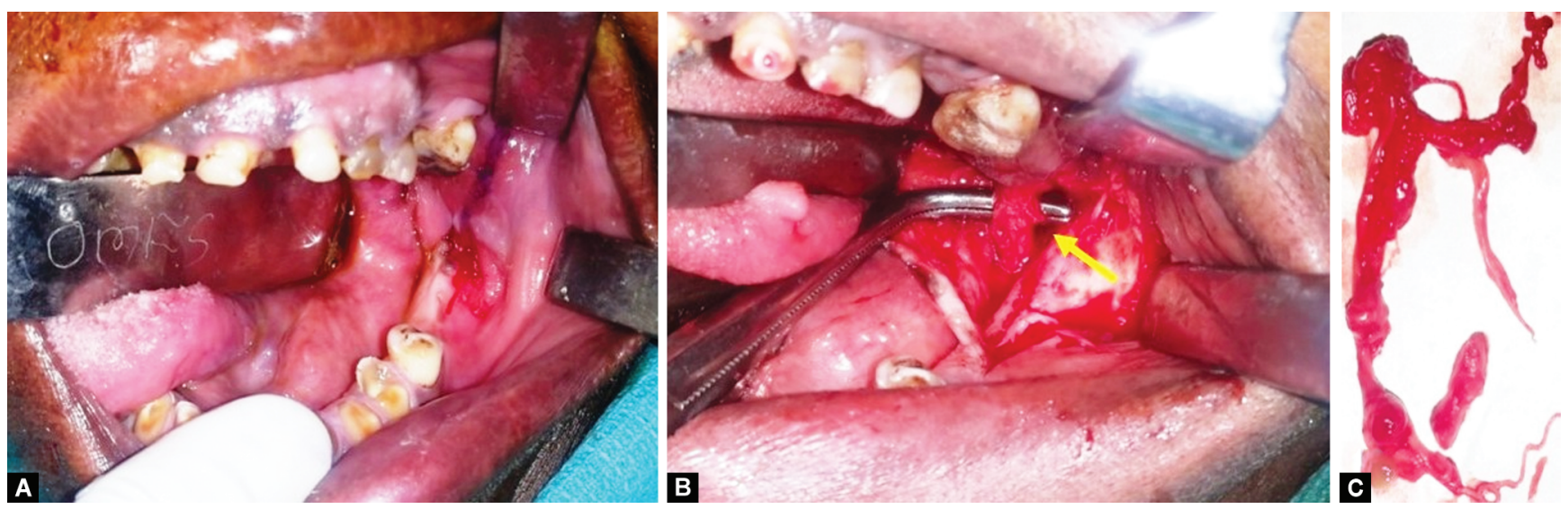

Figs $2 \mathrm{~A}$ to $\mathrm{C}$ : Intraoperative photographs of inferior alveolar nerve neurectomy showing the incision marking at the anterior border of the ramus (A), dissection and exposure of the nerve (yellow arrow) (B), and avulsed nerve (C)
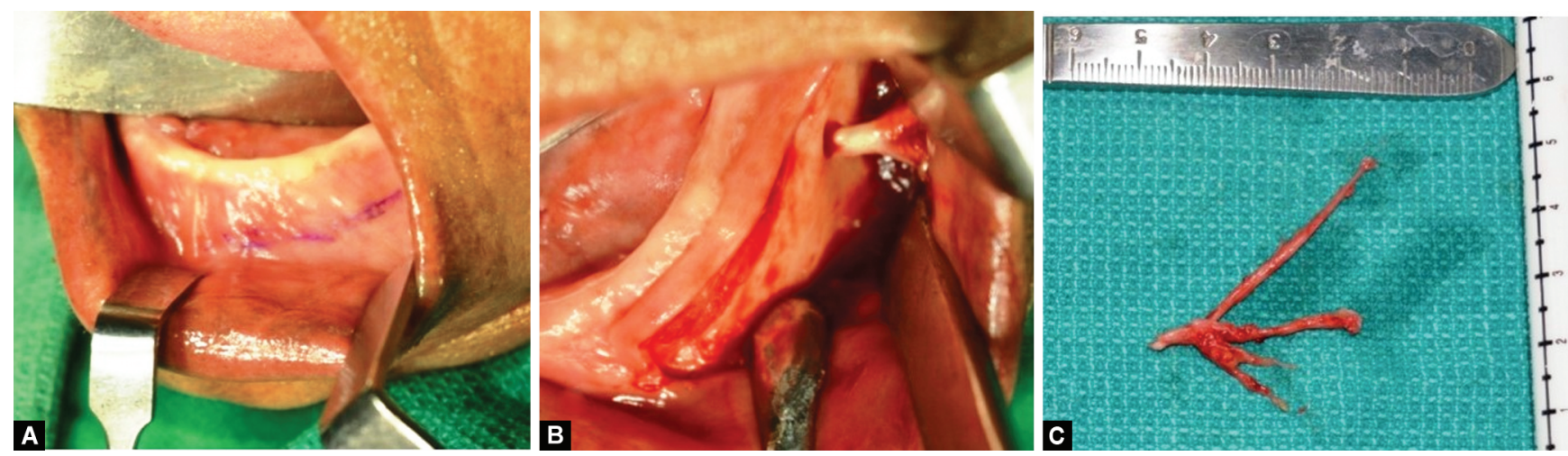

Figs 3 A to C: Intraoperative photographs of mental nerve neurectomy showing the incision marking at lower labial vestibule (A), dissection and exposure of the nerve (B), and avulsed nerve (C)

\section{Supraorbital Nerve Neurectomy}

The supraorbital nerve was approached through an extraoral upper eyebrow incision. Dissection was done to reach the supraorbital ridge. The nerve was identified, freed, and avulsed. The closure was done in layers.

All the patients received postoperative antibiotics and analgesics. The surgical site was subjectively assessed for swelling, bleeding, infection, and wound dehiscence at the 1st postoperative week. Patients were kept on long-term periodic follow-up. Follow-up was scheduled every 6 months, for a minimum of 3 years, to evaluate for recurrence of pain. The follow-up was done by clinical visit or by telephonic interview. Postoperative pain control was assessed using a visual analog scale (VAS), and was graded as good (score of 0-3), fair (score of 4-6), and poor (score of 7-10). Postoperative pain was evaluated postoperatively at the 6th month, 1st year, 2nd year, and 3rd year.

Besides pain, change in the QOL of patients was studied using Short-Form Health Survey (SF-36) questionnaire adapted and validated for the study population. ${ }^{7}$ Short-Form Health Survey is a standard tool to evaluate health-related QOL. It consists of 36 questions, with 8 domains, measuring both the physical and mental components of wellbeing. The physical component is covered by physical functioning (PF), role limitations due to physical health (RP), general health $(\mathrm{GH})$, bodily pain (BP), while the mental component is evaluated by role limitations due to emotional problems (RE), social functioning (SF), mental health (MH) and vitality, energy, and fatigue (VT). The 2 questions of the BP domain were modified to specifically indicate facial pain (FP). Scores of SF-36 questionnaires ranged from 0 to 100 (higher scores indicating better condition). The scores were recorded preoperatively and at the 6th-month, 1st-year, and 3rd-year follow-up.

All the data were entered into MS Excel. All results were calculated using the mean value and standard deviation for each of the parameters considered and checked for statistical significance using the analysis of variance (ANOVA) test. The difference was considered significant if $p<0.05$. The statistical analysis was done by using IBM SPSS ${ }^{\circledR}$ software version 25 (IBM Corp., USA).

\section{Results}

The study was conducted on 32 patients diagnosed with TN, which was refractory to medical (carbamazepine) therapy. Patients who fulfilled the inclusion criteria and were willing for follow-up were included in the study. One patient was lost to follow-up after 6 months and was not included in data analysis. The mean age of patients was 64.48 years, with a range of $55-78$ years (standard deviation 6.08). Both males $(n=10)$ and females $(n=21)$ were enrolled for the study, with male to female ratio of 1:2 (Table 1). The right side of the face was affected in 18 patients (58\%), while the left side was involved in 13 patients (42\%). Patients with refractory TN were on carbamazepine therapy for a period ranging from 6 months to 5 years, with an average of 20.38 months. The dose of carbamazepine ranged from 400 to $1,200 \mathrm{mg} /$ day, with a mean of 
812.90. The reason for termination of medical management was either inability to control pain or intolerance to the drug.

A total of 35 peripheral neurectomies were done in 31 patients. Isolated involvement of mandibular division (V3) of the trigeminal nerve was seen in $58 \%$ of cases $(n=18)$. While only maxillary division (V2) was affected in seven cases (23\%). The first division of the trigeminal nerve was involved in only two patients (6\%). Multiple branches of the trigeminal nerve (V2 + V3) were involved in four patients (13\%). Inferior alveolar nerve was the most frequently involved nerve ( $n=18 ; 51 \%)$, followed by infraorbital ( $n=11 ; 31 \%)$, mental ( $n=4 ; 11 \%)$, and supraorbital nerve ( $n=2 ; 6 \%)$ (Fig. 4).

Out of 31 neurectomies, 4 were done under general anesthesia, while 27 were executed under local anesthesia. Assessment for local complications showed no evidence of wound breakdown, hematoma/ecchymosis, or infection at the 1st postoperative week. Postoperative pain control (success of the surgery) was assessed using VAS. The earliest recurrence of pain was reported at the 6th-month follow-up in one patient. While 27 (87\%) patients were pain-free, $3(10 \%)$ patients required additional low dose carbamazepine ( $200 \mathrm{mg} /$ day) for complete pain control at the 6th-month postoperative period. There was an increase in the number of patients with pain at 1st-year follow-up, with poor and fair control of pain seen in $3(10 \%)$ and 10 (32\%) patients, respectively. At 2nd-year follow-up, 13 patients (42\%) had no pain, while 14 (45\%) required low dose carbamazepine for pain control. Uncontrolled pain was reported by four (13\%) patients. At the final recall visit at 3 years, poor pain control was seen in $32.25 \%$ ( $n=10)$ cases, while an equal number of patients had fair pain control. Eleven patients (35.50\%) had no remission of pain following surgery and did not require any additional medical management (Fig. 5). The mean values of pain score at the follow-up periods of 6 months, 1 year, 2 years, and 3 years were $0.26,1.94,3.52$, and 4.97, respectively. The control of pain was statistically significant, with a $p$

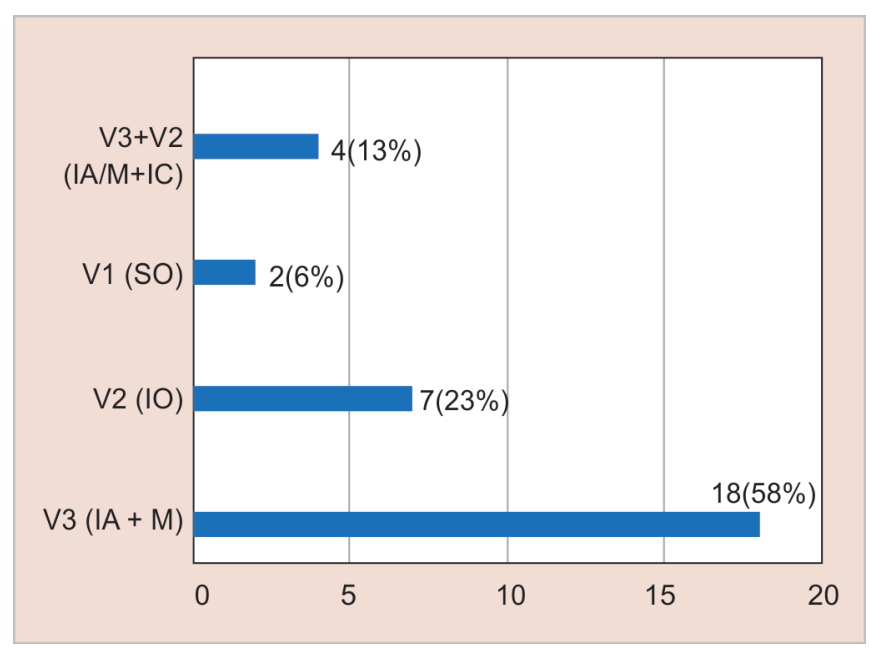

Fig. 4: Graph showing involvement of the three main terminal divisions of the trigeminal nerve

Table 1: Demographic distribution among patients

\begin{tabular}{llll}
\hline & & \multicolumn{2}{c}{ Age (in years) } \\
\cline { 3 - 4 } Gender & Frequency (\%) & Mean & SD \\
\hline Males & $10(32)$ & 66 & 6.28 \\
Females & $21(68)$ & 63.76 & 5.99 \\
\hline
\end{tabular}

value of $<0.0001$ (Table 2). Recurrence of pain after inferior alveolar neurectomy was seen in eight cases (44.44\%), while four patients (36.36\%) reported recurrence of pain after infraorbital neurectomy at the 3-year follow-up.

Evaluation of the physical component of QOL (Table 3) showed an improvement in the preoperative scores of PF, RP, GH, and FP from $38.38 \pm 12.40$ (mean \pm standard deviation), $19.35 \pm 39.5$, $21.61 \pm 11.38$, and $8.38 \pm 12.07$ to $65.64 \pm 8.68,93.54 \pm 21.37$, $81.04 \pm 20.88$, and $88.06 \pm 15.25$ at the 6 th-month follow-up. However, there was a gradual fall in all the physical domains of QOL through the follow-up period, with final scores of $49.03 \pm 17.38$, $58.06 \pm 41.02,53.06 \pm 29.03$, and $57.74 \pm 24.89$ at the 3 rd year after PN (Fig. 6). The mental component of QOL was assessed by measuring $\mathrm{RE}, \mathrm{SF}, \mathrm{MH}$, and $\mathrm{VT}$ (Table 3 ). The respective domains of mental health improved from $4.40 \pm 14.21,14.91 \pm 14.58,15.16 \pm$ 5.54 , and $12.41 \pm 8.64$ at the preoperative period to $94.61 \pm 15.16$, $68.94 \pm 24.76,74.06 \pm 19.51$, and $73.38 \pm 21.61$ at 6 months after PN. However, there was a fall in the scores to $66.63 \pm 31.04,45.56 \pm 27.10$, $47.22 \pm 19.59$, and $42.25 \pm 32.42$ at the 3rd-year follow-up (Fig. 7).

\section{Discussion}

Trigeminal neuralgia is one of the most excruciating pain syndromes afflicting the orofacial region. Over a period of time, various terminologies have been used to describe this disease, including tic-douloureux, Fothergill's disease, and suicide disease. Charles Bell in 1820 first suggested the involvement of the trigeminal nerve and since then it is known as TN. ${ }^{8}$ The characteristic feature of pain in TN is paroxysmal sharpshooting in nature, which is induced by touching the trigger zones over the face. Slight touch or even minor facial movements are known to precipitate severe pain, thereby significantly compromising the function and QOL of the patient.

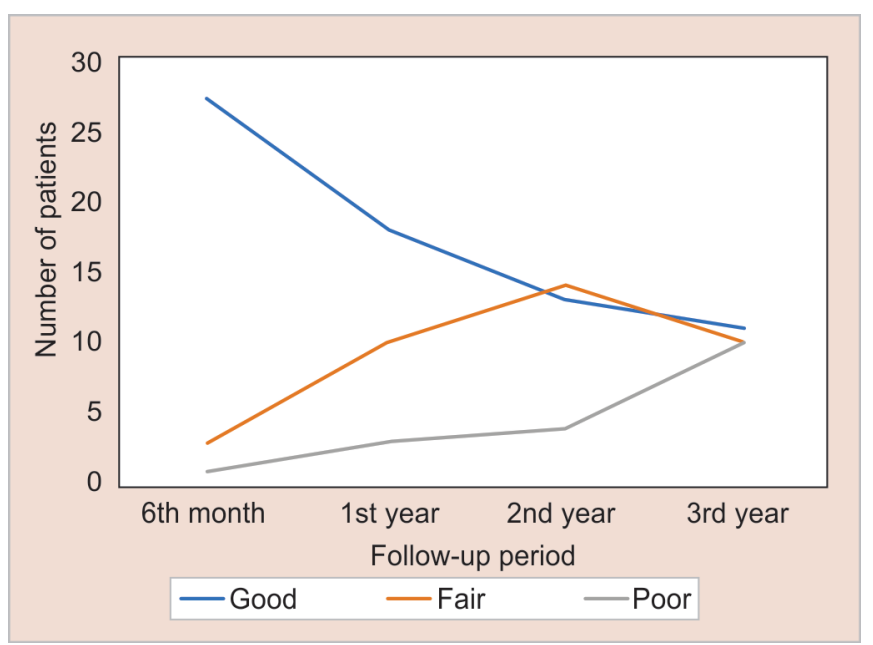

Fig. 5: Graph showing the pain control after peripheral neurectomy at various follow-up periods

Table 2: Pain control after peripheral neurectomy in patients with refractory trigeminal neuralgia

\begin{tabular}{lllll}
\hline & \multicolumn{4}{c}{ Follow-up period } \\
\cline { 2 - 5 } Pain & 6 th months & 1st year & 2nd year & 3rd year \\
\hline Mean & 0.26 & 1.94 & 3.52 & 4.97 \\
SD & 0.82 & 2.7 & 3.11 & 3.91 \\
ANOVA F value & 15.53 & & & \\
$p$ value & $<0.0001$ & & & \\
\hline
\end{tabular}


Table 3: Quality of life (QOL) after peripheral neurectomy in patients with refractory trigeminal neuralgia

\begin{tabular}{|c|c|c|c|c|c|c|c|}
\hline & \multirow[b]{2}{*}{ QOL study variables } & \multicolumn{4}{|c|}{ Time interval } & \multirow[b]{2}{*}{ ANOVA F value } & \multirow[b]{2}{*}{$p$ value } \\
\hline & & Preoperative & 6th month & 1st year & $3 r d$ year & & \\
\hline \multirow[t]{2}{*}{ PF } & Mean & 38.39 & 65.65 & 57.74 & 49.03 & 22.25 & $<0.0001$ \\
\hline & SD & 12.41 & 8.83 & 15.16 & 17.39 & & \\
\hline \multirow[t]{2}{*}{ PR } & Mean & 19.35 & 93.55 & 79.03 & 58.06 & 26.404 & $<0.0001$ \\
\hline & SD & 40.16 & 21.38 & 33.6 & 41.02 & & \\
\hline \multirow[t]{2}{*}{$\mathrm{GH}$} & Mean & 21.61 & 81.05 & 69.35 & 53.06 & 39.313 & $<0.0001$ \\
\hline & SD & 11.58 & 20.88 & 26.19 & 29.04 & & \\
\hline \multirow[t]{2}{*}{ FP } & Mean & 8.39 & 88.06 & 72.58 & 57.74 & 97.56 & $<0.0001$ \\
\hline & SD & 12.27 & 15.26 & 22.58 & 24.89 & & \\
\hline \multirow[t]{2}{*}{ RE } & Mean & 4.4 & 94.62 & 84.93 & 66.64 & 107 & $<0.0001$ \\
\hline & SD & 14.22 & 15.16 & 22.53 & 31.04 & & \\
\hline \multirow[t]{2}{*}{ SF } & Mean & 14.92 & 68.95 & 56.85 & 45.56 & 31.35 & $<0.0001$ \\
\hline & SD & 14.58 & 24.77 & 23.68 & 27.11 & & \\
\hline \multirow[t]{2}{*}{$\mathrm{MH}$} & Mean & 15.16 & 74.06 & 62.13 & 47.23 & 65.04 & $<0.0001$ \\
\hline & SD & 5.46 & 19.51 & 21.07 & 19.59 & & \\
\hline \multirow[t]{2}{*}{ VT } & Mean & 12.42 & 73.39 & 59.19 & 42.26 & 32.3 & $<0.0001$ \\
\hline & SD & 8.65 & 21.62 & 32.36 & 32.43 & & \\
\hline
\end{tabular}

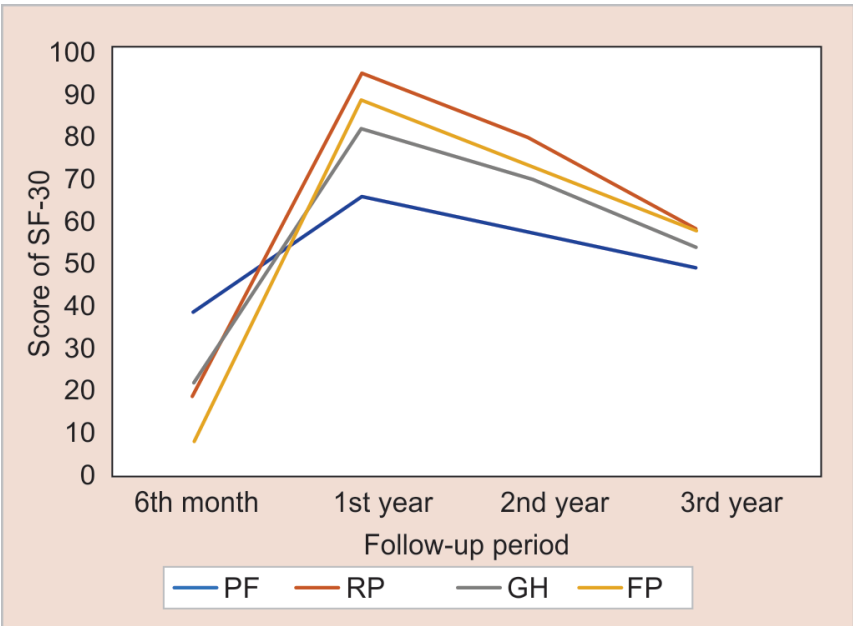

Fig.6: Graph showing SF-36 scores for physical components at different time intervals after PN

Although various surgical treatments are available, pharmacological agents are initially used for management for $\mathrm{TN} .{ }^{9}$ Efficacy of carbamazepine and/or oxcarbazepine as the first choice of drug in the treatment of TN is well established..$^{10}$ They are used in a dose of 200-1,200 and 600-1,800 mg/day, respectively. However long-term use of these anti-epileptic drugs is associated with various complications, including dizziness, sedation, nausea, ataxia, hyponatremia, leukopenia, aplastic anemia, allergic reaction, hepatotoxicity, and systemic lupus erythematosus, leading to intolerance and reduced patient compliance. ${ }^{11,12}$ Hence, it is recommended to get complete blood count, liver function tests, and serum sodium levels monitored, during the therapy. Another limitation of carbamazepine is the development of resistance to the drug and recurrence of pain. In a study of long-term treatment of TN with carbamazepine in 143 patients, Taylor et al. reported severe side effects requiring cessation of the therapy in $6 \%$ of cases, while $13 \%$ had a recurrence of pain between 2 months and 10 years, with

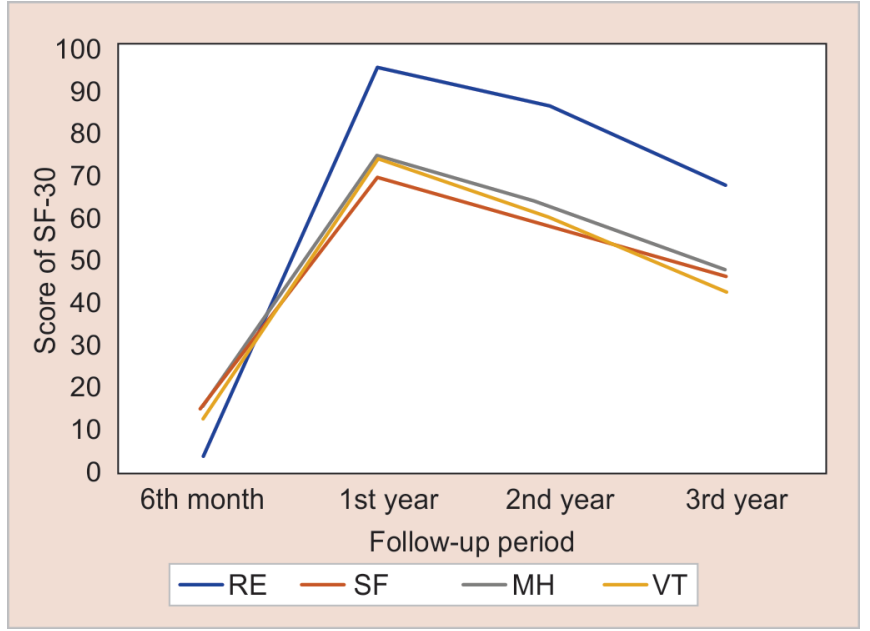

Fig. 7: Graph showing SF-36 scores for mental components at different time intervals after PN

a mean of 4 years after starting the drug. ${ }^{13}$ Although various other antiepileptic drugs including baclofen, gabapentin, and pregabalin have been used, there is limited evidence of their efficacy to treat TN. ${ }^{14}$ In the present study, the patients with refractory TN were on carbamazepine therapy for a period of 6 months to 5 years (mean 20.38 months), with the dose ranging from 400 to 1,200 $\mathrm{mg} /$ day (mean dose of $800 \mathrm{mg} /$ day). According to the literature, TN reportedly become refractory to carbamazepine therapy after a period ranging from 8 to 72 months. ${ }^{15}$

According to the European Federation of Neurological Society (EFNS) and the American Academy of Neurology (AAN) guidelines, patients who are intolerant or non-responsive to carbamazepine therapy should be treated surgically. ${ }^{16}$ Various surgical modalities are available for the management of TN and include microvascular decompression, percutaneous glycerol or radiofrequency rhizotomy, stereotactic radiosurgery, and percutaneous balloon compression. ${ }^{17}$ Microvascular decompression remains the gold 
standard, among all the procedures with reported relief of pain in up to $80 \%$ of cases at the 1-year follow-up. ${ }^{1}$ However, it is an invasive surgery requiring craniotomy to access the intracranial part of the trigeminal nerve. Recurrence of pain after microvascular decompression has been reported in about $25 \%$ of cases on longterm follow-up. ${ }^{18}$ Serious complications are associated with $4 \%$ of cases and include cerebrospinal fluid leakage, infarcts/hematoma, meningitis, sensory, and hearing loss. The average mortality rate in microvascular decompression surgery ranges from 0.2 to $0.5 \%{ }^{14}$ Cranial surgeries are associated with a longer hospital stay, higher cost of treatment, and greater risk of complications. In contrast, the peripheral procedures, including $\mathrm{PN}$, alcohol injection, and cryotherapy, are relatively simple and less invasive. However, these procedures have been associated with varying success rates. Choice of surgical procedure is dependent both on patients' physical and mental condition, and the preference of the surgeon and available infrastructure. Zakrzewska et al. stated that patient's participation in the decision-making for choice of treatment of TN is very important. ${ }^{19}$ Some patients may desire long-term pain relief at cost of higher risk of morbidity and treatment expense, while others may accept short-term pain relief in return for fewer complications. In a survey in the United Kingdom, when oral and maxillofacial surgeons were questioned about the management of TN refractory to medical therapy, equal numbers responded on the choice between peripheral procedures and referral to a neurosurgeon. ${ }^{20}$

Peripheral neurectomy is a simple surgery that involves avulsion of the affected terminal branch of the trigeminal nerve. This procedure was first reported in the year 1830 and since then various reviews and studies have been published evaluating its efficacy and utility in the management of TN. ${ }^{21}$ The main advantage of PN is that it is less invasive and is therefore associated with minimal complications as compared to central procedures. No local complication was seen in the 35 neurectomies performed in the present study. The fact that most of the neurectomies were performed in an outpatient setting and under local anesthesia, avoided risk in susceptible patients in whom major surgery or general anesthesia was contraindicated. Out of 31 patients who underwent neurectomies, 4 involving inferior alveolar nerve were performed under general anesthesia (13\%). In literature, PN has been used for all the terminal branches of the trigeminal nerve including infraorbital, mental, lingual, infraorbital, supraorbital, supratrochlear, and lacrimal nerves. ${ }^{22}$ Inferior alveolar (51\%) and infraorbital (31\%) nerves were most frequently involved in the present study.

Although PN is a relatively inexpensive procedure with low morbidity, a higher rate of recurrence of pain has limited its popularity for the management of TN. Most of the studies on PN for the management of refractory TN have been retrospective data analysis, with a varying period of follow-up and recurrence. In one of the longest analyses of 60 months, Khanna and Galinde reported recurrence of pain in $25 \%$ of cases. ${ }^{23}$ Lamichhane et al. had a recurrence in $35 \%$ of cases, after 26 months of PN surgery. ${ }^{24}$ Study by Grantham and Segerberg showed a mean painless period of 33.2 months after 55 neurectomies. ${ }^{25}$ While Quinn and Weil operated on 88 patients with refractory TN and documented pain relief for an average period of 41 months after PN. ${ }^{26}$ In the present study, good pain relief was seen initially after PN, with poor pain control seen in only 1 (3.22\%) and 3 (9.67\%) patients at 6th month and 1 year of follow-up, respectively. However, 3 years after the procedure, 11 (35.48\%) patients had fair and 10 (32.25\%) patients had poor control of pain. There is no consensus in the literature on the relation between the terminal branch of the trigeminal nerve and the success of PN. While Khanna and Galinde reported a greater pain-free period after mental nerve neurectomy, Mason observed that neurectomy of infraorbital neurectomy was more successful than inferior alveolar nerve. ${ }^{23,27}$ In this study, at the 3-year follow-up, inferior alveolar and infraorbital neurectomy were associated with pain in 44.44 and $36.36 \%$ of cases, respectively. Recent studies have recommended packing the mental and infraorbital nerve foramen with various materials like a metal screw, fat, gold foils, and silicone to reduce the risk of recurrence. Occlusion of the foramen is believed to retard nerve regeneration, thereby reducing relapse of pain. ${ }^{28}$

Trigeminal neuralgia is known to be a severely debilitating disease. The pain is invariably so intense that it affects the general wellbeing of an individual. Hence, the treatment for TN must not only aim at controlling pain but also improve the QOL of the individual. Although few studies have reported the effect of central procedures on the QOL in patients with $\mathrm{TN}$, assessment of $\mathrm{PN}$ on QOL has not been done before. In the present study, the QOL of patients with refractory TN was studied using SF-36 questionnaire. The SF-16 is a standard tool with eight domains that measure both the physical and mental wellbeing of the patient. Peripheral neurectomy resulted in a marked improvement on $\mathrm{QOL}$ in the early follow-up period of 6 months and 1 year. Although there was a fall in the mean SF-36 scores at long-term follow-up of 3 years, it was above the preoperative baseline value, indicating an improved QOL.

\section{ConCLUSion}

The choice of management of refractory TN is various and can be broadly grouped under central and peripheral procedures. Although most of the central procedures provide pain control for a longer period of time, they are associated with a greater cost and higher morbidity. Microvascular decompression and partial rhizotomy are major neurosurgical interventions that carry a higher risk of death and morbidity. Radiofrequency thermocoagulation results in an extensive sensory loss. Although PN is associated with relapse, it provided good to fair control of pain in $65 \%$ of cases, 3 years after the procedure. An appreciable improvement in QOL was found, which despite the decrease was considerably above the preoperative baseline on long-term follow-up. Although PN has limited indications, it is a valuable option in patients who are not willing for higher surgical risk or not fit for major surgical intervention under general anesthesia. It is also a useful treatment option in a rural setting with limited resources. However, due to a limited number of patients enrolled, a future study design recruiting a larger number of patients and with a longer follow-up duration can provide further information on the efficacy of PN in patients with refractory $\mathrm{TN}$.

\section{Author Contributions}

Investigator, drafting, and final approval of the manuscript.

\section{References}

1. Obermann M. Treatment options in trigeminal neuralgia. Ther Adv Neurol Disord 2010;3(2):107-115. DOI: 10.1177/1756285609359317.

2. Yadav YR, Nishtha Y, Sonjjay P, et al. Trigeminal neuralgia. Asian J Neurosurg 2017;12(4):585-597. DOI: 10.4103/ajns.AJNS_67_14.

3. Miller JP, Acar F, Burchiel KJ. Classification of trigeminal neuralgia: clinical, therapeutic, and prognostic implications in a series of 144 patients undergoing microvascular decompression. J Neurosurg 2009;111(6):1231-1234. DOI: 10.3171/2008.6.17604. 
4. Campbell FG, Graham JG, Zilkha KJ. Clinical trial of carbazepine (tegretol) in trigeminal neuralgia. J Neurol Neurosurg Psychiatry 1966;29(3):265-267. DOI: 10.1136/jnnp.29.3.265.

5. Tatli M, Satici O, Kanpolat $Y$, et al. Various surgical modalities for trigeminal neuralgia: literature study of respective long-term outcomes. Acta Neurochir (Wien) 2008;150(3):243-255. DOI: 10.1007/ s00701-007-1488-3.

6. Montano N, Papacci F, Cioni B, et al. What is the best treatment of drug-resistant trigeminal neuralgia in patients affected by multiple sclerosis? A literature analysis of surgical procedures. Clin Neurol Neurosurg 2013;115(5):567-572. DOI: 10.1016/j.clineuro.2012. 07.011.

7. Montano N, Conforti G, Bonaventura R, et al. Advances in diagnosis and treatment of trigeminal neuralgia. Ther Clin Risk Manag 2015;11:289-299. DOI: 10.2147/TCRM.S37592.

8. Sinha R, van den Heuvel WJ, Arokiasamy P. Validity and reliability of MOS short form health survey (SF-36) for use in India. Indian J Community Med 2013;38(1):22-26. DOI: 10.4103/0970-0218. 106623.

9. Al-Quliti KW. Update on neuropathic pain treatment for trigeminal neuralgia: the pharmacological and surgical options. Neurosciences (Riyadh) 2015;20(2):107-114. DOI: 10.17712/nsj.2015.2.20140501.

10. Cruccu G, Gronseth G, Alksne J, et al. AAN-EFNS guidelines on trigeminal neuralgia management. Eur J Neurol 2008;15(10):10131028. DOI: 10.1111/j.1468-1331.2008.02185.x.

11. Besi E, Boniface DR, Cregg R, et al. Comparison of tolerability and adverse symptoms in oxcarbazepine and carbamazepine in the treatment of trigeminal neuralgia and neuralgiform headaches using the Liverpool adverse events profile (AEP). The J Headache Pain 2015;16(1):563. DOI: 10.1186/s10194-015-0563-z.

12. Hassan S, Khan NI, Sherwani OA, et al. Trigeminal neuralgia: an overview of literature with emphasis on medical management. Int Research J Pharmcol 2013;3:235-238.

13. Taylor JC, Brauer S, Espir ML. Long-term treatment of trigeminal neuralgia with carbamazepine. Postgrad Med J 1981;57(663):16-18. DOI: 10.1136/pgmj.57.663.16.

14. Gronseth G, Cruccu G, Alksne J, et al. Practice parameter: the diagnostic evaluation and treatment of trigeminal neuralgia (an evidencebased review): report of the quality standards subcommittee of the American Academy of Neurology and the European Federation of Neurological Societies. Neurology 2008;71(15):1183-1190. DOI: 10.1212/01.wnl.0000326598.83183.04.
15. Agrawal SM, Kambalimath DH. Peripheral neurectomy: a minimally invasive treatment for trigeminal neuralgia. A retrospective study. J Maxillofac Oral Surg 2011;10(3):195-198. DOI: 10.1007/s12663-0110229-6.

16. Cruccu G, Andrea Truini A. Refractory trigeminal neuralgia nonsurgical treatment options. CNS Drugs 2013;27(2):91-96. DOI: 10.1007/ s40263-012-0023-0.

17. Bick SKB, Eskandar EN. Surgical treatment of trigeminal neuralgia. Neurosurg Clin N Am 2017;28(3):429-438. DOI: 10.1016/j. nec.2017.02.009.

18. Teive HA, Piovesan EJ, Kowacs PA, et al. Comment on AAN-EFNS guidelines on trigeminal neuralgia management. Eur J Neurol 2009;16(5):e105. DOI: 10.1111/j.1468-1331.2009.02584.x.

19. Zakrzewska JM. Surgical management of trigeminal neuralgia. $\mathrm{Br}$ Dent J 1991;170(2):61-62. DOI: 10.1038/sj.bdj.4807418.

20. McLeod NMH, Tekeli KM, Cheriyan J. Trigeminal neuralgia: assessment and management by oral and maxillofacial surgeons in the United Kingdom. Br J Oral Maxillofac Surg 2009;47(1):42-45. DOI: 10.1016/j. bjoms.2008.06.007.

21. Wilkins RH. Historical overview of surgical techniques for trigeminal neuralgia. Tech Neurosurg 1999;5(3):202-217. DOI: 10.1097/00127927199905030-00003.

22. Rubin W. Treatment of first-division Tic Douloureux by peripheral neurectomy. AMA Arch Ophthalmol 1958;59(2):243-246. DOI: 10.1001/ archopht.1958.00940030111011.

23. Khanna JN, Galinde JS. Trigeminal neuralgia report of 140 cases. Int J Oral Surg 1985;14(4):325-332. DOI: 10.1016/s0300-9785(85)80021-1.

24. Lamichhane NS, Du X, Li S, et al. Effectiveness of peripheral neurectomy in refractory cases of trigeminal neuralgia. J Orofac Sci 2016;8(2):86-91. DOI: 10.4103/0975-8844.195908.

25. Grantham E, Segerberg LH. An evaluation of palliative surgical procedures in trigeminal neuralgia. J Neurosurg 1952;9(4):390. DOI: 10.3171/jns.1952.9.4.0390.

26. Quinn JH, Weil T. Trigeminal neuralgia: treatment by repetitive peripheral neurectomy. Supplemental report. J Oral Surg 1975;33(8):591-595.

27. Mason DA. Peripheral neurectomy in the treatment of trigeminal neuralgia of the second and third divisions. J Oral Surg 1972;30(2):113120.

28. Ali FM, Prasant MC, Pai D, et al. Peripheral neurectomies: a treatment option for trigeminal neuralgia in rural practice. J Neurosci Rural Pract 2012;3(2):15-17. DOI: 10.4103/0976-3147.98218. 